RATING-BERATUNG

\title{
Fit für den Markt
}

\author{
Hubert Oppl und Ulrike Pech
}

\begin{abstract}
Die Finanzmarktkrise erschwert die durch »Basel II «schon verschärften Kreditbedingungen für Unternehmen der Sozialwirtschaft noch weiter. Eine externe Beratung und ein unabhängiges Rating können helfen, die Finanzzierungsbedingungen zu erleichtern.
\end{abstract}

In der Diskussion um die Pflegeversicherung wurde und wird ein Thema übersehen: die Finanzierung der Investitionen. Die Einführung der Pflegeversicherung folgte in erster Linie dem Ziel, die Sozialhilfeträger von den laufenden Kosten der stationären Pflege zu entlasten. Zur Kompensation haben sich die überörtlichen Träger der Sozialhilfe zur Förderung der Investitionen verpflichtet. In den vergangenen Jahren haben diese sich jedoch Schritt für Schritt aus dieser Aufgabe zurückgezogen, auch aus der Förderung von Instandhaltungsinvestitionen. Dieser Rückzug der öffentlichen Hände aus der Förderung traf in erster Linie die gemeinnützigen Betreiber. Für sie bestehen mindestens drei Probleme:

- Ihre bisherige durch das Gemeinnützigkeitsprivileg geschützte Stellung führte zu einer »Markabstinenz «. In aller Regel fehlt diesen Betreibern Markterfahrung sowie Erfahrung im Umgang mit dem Kapitalmarkt.

- Preisvergleiche zeigen, dass die Pflegesätze geförderter Häuser oft teurer sind als frei finanzierte. Bei einer Vollkostenkalkulation würden die Kosten weiter steigen und die Position dieser Häuser im Wettbewerb wird gefährdet.

- Bisherige eher an Bedarfsgesichtspunkten orientierte Angebote verhinderten die Auseinandersetzung mit Konkurrenz und Wettbewerb sowie eine daran orientierte Gestaltung von Leistungen und Preisen.
Erschwerend kommen ungünstige unternehmerische Zuschnitte hinzu. Vielfach sind Besitz- sowie Betriebsgesellschaft identisch, die »Produktionstiefe zu intensiv.

Eine alternative Finanzierung über den Kapitalmarkt ist an eine Vielzahl von Voraussetzungen geknüpft, die gemeinnützige Vereine und ihre Zweckbetriebe gar nicht erfüllen (können). Neben »wettbewerbstauglichen « organisatorischen und betrieblichen Zuschnitten fehlt den gemeinnützigen Betreibern in aller Regel eine hinreichende Eigenkapitalausstattung.

Investoren und Banken tun sich nach wie vor mit dem Sozialmarkt schwer, besonders aber mit seinen gemeinnützigen Akteuren. Da sich bankenübliche Bewertungsverfahren nur begrenzt auf sozialwirtschaftliche Unternehmen - insbesondere Pflegeheime - übertragen lassen, haben Branchenbanken eigene Ratingverfahren entwickelt. Neben dem bankinternen Rating bietet es sich jedoch auch an, auf unabhängige externe Ratingpartner zurückzugreifen.

Das Finanzierungsgeschäft hat sich in den vergangenen Jahren $\mathrm{v}$ nicht zuletzt als Folge von Fehleinschätzungen und dem in der Folge entstandenen Verhaltenskodex Basel II erheblich gewandelt. Die Bonität eines Kunden muss nicht allein umfänglich geprüft werden, sie hat nachhaltigen Einfluss auf die Finanzierungskonditionen. Sämtliche Organisationen und Unternehmen, die einen Kredit beantragen, werden sich einem bankinternen Rating stellen müssen. Die Prüfungen werden dabei detaillierter, tiefer und genauer erfolgen, als dies in der Vergangenheit der Fall war.

Der Wandel in der Gestaltung und der Finanzierung von Investments in der Sozialwirtschaft folgend, müssen sich auch Organisationen und Unternehmen der Sozialwirtschaft einem entsprechenden Bankenrating stellen. In Vorbereitung darauf sollten die jeweiligen Unternehmen durch ein entsprechendes externes Rating, verbunden mit einem Rating Advisory die Möglichkeiten nutzen, dem bankinternen Rating ein externes gegenüberzustellen. Damit kann gegebenenfalls das bankinterne Rating verbessert werden.

\section{Externes Rating}

Bei einem externen Rating wird die Kundenbonität durch einen unabhängigen Dritten beurteilt. Dies kann im Auftrag des Kapitalgebers stattfinden oder auch im Auftrag des Kunden in Vorbereitung auf ein internes Rating des Finanzierungsinstitutes.

Der Ratingprozess stützt sich dabei auf ein objektives, vergleichbares als Ratingsystem ausgebildetes Verfahren, das auf operationalisierte Erhebungsinstrumente und Erhebungsschritte aufbaut. Sie bestehen in aller Regel in einem Vorgespräch, in dem die Kriterien, die erforderlichen Unterlagen sowie die spezifischen Methoden erörtert werden. Darauf aufbauend bereitet die Agentur ein Hauptgespräch oder ManagementMeeting vor. Die Ergebnisse des Meetings, das sich häufig auf ein bis zwei Tage konzentriert, werden von der Agentur analysiert und um weitere markt- sowie branchenspezifische allgemeine Daten (Branche, Markt, Marktumfeld, Konkurrenz, Produktbenchmarks usw.) ergänzt. Die ermittelten Daten sowie die gewonnenen Informationen werden in das Ratingsystem der Agentur übertragen und ausgewertet.

\section{Beurteilungskriterien}

Abhängig von der jeweiligen Ratingagentur variiert die Auswahl der Beurteilungskriterien je nach Branchen und Zielsetzungen des Ratings. Sie ähneln sich letztendlich in den Indikatoren und ihren Grundaussagen. Wesentlich ist, dass bei der Auswahl und Festlegung der Kriterien die Besonderheiten der Sozialwirtschaft, wie ihr Unternehmensaufbau und ihre Finanzierungsstruktur, berücksichtigt und aufbereitet werden, welche häu- 
fig bei klassischen Ratings der Banken zu einem negativen Bonitätsurteil führen würden. Für Unternehmen der Sozialwirtschaft werden grundsätzlich drei Analysefelder betrachtet: wirtschaftliche Verhältnisse, qualitative Unternehmensbewertung und eine Branchen- und Umfeldanalyse.

\section{Analysefeld wirtschaftliche Verhältnisse}

Im Feld der wirtschaftlichen Verhältnisse werden im klassischen Sinne die Vermögens-, Finanz- und Ertragslage des Unternehmens bewertet. Weiterhin werden aber auch die historische sowie die geplante Entwicklung bei der Beurteilung mit einbezogen. So werden beispielsweise prospektive Aspekte und Instrumente wie die Existenz einer Liquiditätsplanung sowie die Durchführung von Planungsprozessen betrachtet.

\section{Analysefeld qualitative Unternehmensbewertung}

Während sich die Beurteilung der wirtschaftlichen Verhältnisse vornehmlich auf quantitative Kriterien stützt, sollen Indikatoren des Analysefeldes »qualitative Unternehmensbewertung « Informationen darüber liefern, wie der Unternehmenszuschnitt gestaltet und ob er den gegenwärtigen und künftigen Marktanforderungen gewachsen ist. Bei diesem Analysefeld werden $u$. a. Indikatoren $\mathrm{zu}$ folgenden Bereichen erhoben und bewertet:

- Unternehmensstruktur und Unternehmensgliederung

- Organisation und Führungsstruktur

- Managementqualität

- Rechnungswesen und Controlling

- Mitarbeiterstruktur und Mitarbeiterqualität

Die Untersuchung und Bewertung dieser qualitativen Parameter liefern eine sichere Grundlage für die Beurteilung der Leistungsfähigkeit, der Managementkompetenz und damit der operativen Kompetenz der analysierten Organisation sowie ihrer Zweckbetriebe.

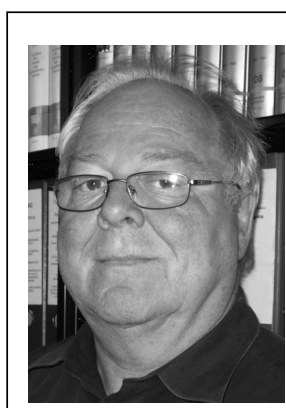

Prof. Dr. Oppl ist Diplom-Sozialpädagoge und war bis 1997 an der Katholischen Stiftungsfachhochschule München Hochschullehrer für Sozialarbeit, Pädagogik und Unternehmensführung in der Sozialwirtschaft. Managementerfahrungen bei sozialwirtschaftlichen Unternehmen sammelte er dann bei den Franziskanerinnen von Reute (ca. 2.500 Mitarbeiter), Oberschwabenkliniken (7 Kliniken im Verbund) sowie der Refugium AG. Danach machte er sich mit mehreren Unternehmen selbstständig. Schwerpunkte seiner Beratungen für die Sozialwirtschaft sind Finanzierungslösungen für Sozialimmobilien sowie Lösungen der betrieblichen Altersvorsorge in der Sozialwirtschaft. E-Mail hubert.oppl@carefuture.de

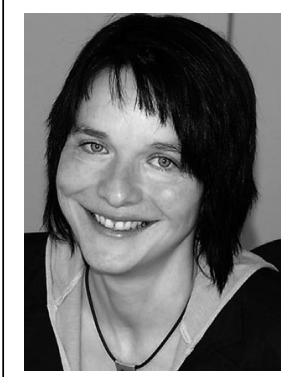

Prof. Dr. Ulrike Pech ist Hochschullehrerin im Wirtschaftsbereich an der Staatlichen Studienakademie Thüringen, Berufsakademie Gera. Sie vertritt die Fächer Rechnungswesen (Buchführung, Kosten- und Leistungsrechnung), Bilanzierung, Steuern und Controlling und ist Studienrichtungsleiterin Handel. Sie studierte Betriebswirtschaftslehre und Wirtschaftspädagogik an der Friedrich-Schiller-Universität Jena und arbeitete dann als Abteilungsleiterin Wirtschaft bei einer gemeinnützigen Trägergesellschaft mit 700 Mitarbeitern. Im Jahr 2000 promovierte sie an der Universität Jena zum Thema Bildungscontrolling. Danach arbeitete sie als Controllerin bei einem Sozialversicherungsträger und als Dozentin für Rechnungswesen, Bildungsmanagement sowie als freiberufliche Controllerin. Seit 2005 ist sie an der Berufsakademie beschäftigt.

E-Mail ulrike.pech@ba-gera.de

\section{Analysefeld Branche und Umfeld}

In der Branchen- und Umfeldanalyse liegt ein besonderes Gewicht des Ratings. Zu den Kernkriterien zählen u. a. Informationen über den Markt, Standorte, Service, allgemeine und lokale Wettbewerbssituation, Marktpotenzial, das Qualitätsmanagement sowie Chancen und Risiken der Geschäftstätigkeit.

Aufbauend auf die Erfassung der Einzelkriterien sowie Bewertung ihrer jeweiligen Ausprägungen werden diese jeweils gewichtet und innerhalb des jeweiligen Analysefeldes zu einer Feldnote zusammengefasst. Die sich daraus ableitenden drei Feldnoten werden dann nochmals gewichtet und zu einer Gesamtnote zusammengefasst. Aus dieser leitet sich abschließend die Gesamteinschätzung ab. Sie kann mit Hinweisen zur Verbesserung der Ratingnote durch gezielte Reorganisationsschritte verbunden werden.

\section{Wie man sein Rating verbessern kann}

Da vielfach sozialwirtschaftliche Unternehmen sowie ihre Organisationen nicht oder nicht hinreichend fit für den Markt und Drittfinanzierungen sind, bietet es sich an, begleitend zu einem Rating - ihm vorausgehend oder auch folgend - eine Ratingberatung durchzuführen. Ziel dieser Ratingberatung ist es, ein für die künftigen Ziele des Unternehmens optimalen Unternehmenszuschnitt und darauf aufbauend ein optimales Rating zu erreichen. Aus Sicht der beauftragenden Organisation dient ein ergänzendes Coaching im Vorfeld eines Ratings der effektiven und effizienten Durchführung des Ratingverfahrens.

Der Advisor baut die Analysetätigkeit im Sinne einer StärkenSchwächen-Analyse in Hinblick auf die Ratingkriterien auf. Ihr Ziel konzentriert sich auf die Ermittlung mög- 
licher Schwachstellen, die Ableitung von Vorschlägen und gegebenenfalls Umsetzung von Verbesserungsmaßnahmen im Hinblick auf das anschließende Ratingverfahren. Der Advisor steht auch für die Koordination und Leitung der jeweiligen Projektteams zur Verfügung, welche die anstehenden Reorganisationsaufgaben umsetzen müssen.

Ergebnis kann unter anderem die Simulation eines Ratingverfahrens sein, anhand dessen nochmals wesentliche Strategien und Maßnahmen erarbeitet werden. Letztendlich kann der Rating Advisor auch unterstützend beim Ratinggespräch mit dem Kapitalgeber tätig werden und damit eine bessere Verhandlungsposition und ein besseres Verhandlungsergebnis ermöglichen.

Beide Möglichkeiten - externes Rating und Rating Advisory - können getrennt voneinander, aber auch kombiniert angeboten und durchgeführt werden. Sie nützen der Organisation sowohl im Hinblick auf die Finanzierungszusage als auch in Hinblick auf die Durchsetzung günstigerer Finanzierungskonditionen. Bei einem Finanzierungsvolumen in Höhe beispielsweise von zehn Millionen Euro kann ein halber oder ganzer Prozentpunkt weniger Zinsen schnell einen Einsparungsbetrag von über 100.000 Euro ergeben. Dies rechtfertigt sicherlich den Aufwand für begleitende Ratingdienstleistungen.

\section{Literatur:}

KfW Bankengruppe (Hg): Rating für den Mittelstand. Wissen, worauf es ankommt., Frankfurt am Main 2003.

Hückmann: Kreditrating der Mittel- und Kleinbetriebe. Eine Orientierungs- und Entscheidungshilfe zu den Verfahren der Kreditinstitute und Agenturen, 2. Auflage. Berlin 2003.

DIHK (Hg): Rating für den Mittelstand. Fit für das Gespräch mit der Hausbank, Berlin 2003.

\section{Expertenwissen für Führungskräfte}

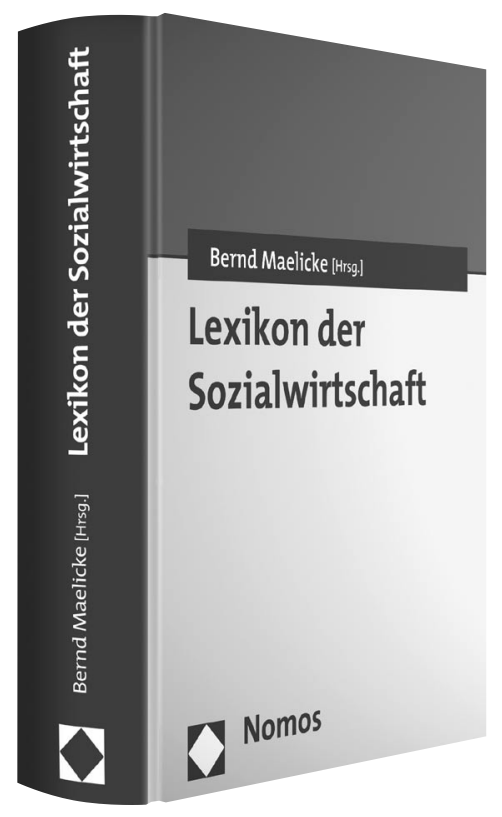

Lexikon der

Sozialwirtschaft

Herausgegeben von Prof. Dr. Bernd Maelicke 2007, 1.128 S., geb., 98,-€, ISBN 978-3-8329-2511-6

"Verdienstvoll und gelungen...Ein qualitätsvolles Handbuch mit
Zukunft."
Neue Caritas 11/08

"Es ist das erste Überblickswerk zu der Thematik und stellt auf 1.128 Seiten dar, was gegenwärtig fachlich und sachlich der Stand des Wissens zu sozialwirtschaftlichen Fragen ist."

Prof. Dr. Wolf Rainer Wendt, Zeitschrift für Betreuungsmanagement 69/08

"Es ist das erste Überblickswerk zu der Thematik und stellt auf 1.128 Seiten dar, was gegenwärtig fachlich und sachlich der Stand des Wissens zu sozialwirtschaftlichen Fragen ist."

Prof. Dr. Wolf Rainer Wendt, Zeitschrift für Betreuungsmanagement 69/o8

"Ein exzellentes Nachschlagewerk für den Bereich der Sozialen Arbeit und den angrenzenden Arbeitsfeldern...ein zu empfehlendes Nachschlagewerk für all jene, die fundierte Informationen schnell auffinden müssen und dabei einen wissenschaftlichen Anspruch haben."

Tarek Bamberger, Bonn, www.sozialarbeitsnetz.de, Nov. 2007 